\title{
Redshift constraints for RGB 0136+391 and PKS 0735+178 from deep optical imaging ${ }^{\star}$ (Research Note)
}

\author{
K. Nilsson ${ }^{1}$, T. Pursimo ${ }^{2}$, C. Villforth ${ }^{3}$, E. Lindfors ${ }^{4}$, L. O. Takalo ${ }^{4}$, and A. Sillanpää ${ }^{4}$ \\ ${ }^{1}$ Finnish Centre for Astronomy with ESO (FINCA), University of Turku, Väisäläntie 20, 21500 Piikkiö, Finland \\ e-mail: kani@utu.fi \\ 2 Nordic Optical Telescope, Apartado 474, 38700 Santa Cruz de La Palma, Spain \\ 3 Department of Astronomy, University of Florida, 211 Bryant Space Science Center, Gainesville, FL 32611-2055, USA \\ 4 Tuorla Observatory, Department of Physics and Astronomy, University of Turku, Väisäläntie 20, 21500 Piikkiö, Finland
}

Received 20 June 2012 / Accepted 13 September 2012

\section{ABSTRACT}

\begin{abstract}
We present the results of deep I-band imaging of two BL Lacerate objects, RGB 0136+391 and PKS 0735+178, during an epoch when the optical nucleus was in a faint state in both targets. In PKS $0735+178$ we find a significant excess over a point source, which, if fitted by the de Vaucouleurs model, corresponds to a galaxy with $I=18.64 \pm 0.11$ and $r_{\mathrm{eff}}=1.8 \pm 0.4$ arcsec. Interpreting this galaxy as the host galaxy of PKS $0735+178$ we derive $z=0.45 \pm 0.06$ using the host galaxy as a "standard candle". We also discuss the immediate optical environment of PKS $0735+178$ and the identity of the MgII absorber at $z=0.424$. Despite of the optimally chosen epoch and deep imaging we find the surface brightness profile of RGB $0136+391$ to be consistent with a point source. By determining a lower limit for the host galaxy brightness by simulations, we derive $z>0.40$ for this target.
\end{abstract}

Key words. galaxies: active - galaxies: nuclei - BL Lacertae objects: individual: RGB 0136+391 -

BL Lacertae objects: individual: PKS 0735+178

\section{Introduction}

The optical spectrum of BL Lacertae objects (BL Lacs) is dominated by a featureless synchrotron continuum, which makes the determination of their redshift by spectroscopy very challenging. Redshift determination of quasars and BL Lacs is usually based on emission lines originating in the narrow and broad line regions and/or on the absorption features of the host galaxy. However, in BL Lacs the emission lines are intrinsically weak and like the host galaxy absorption lines they often become so weak relative to the continuum that they fall below the noise level and become unrecognizable.

It is therefore not surprising that many BL Lac samples still have a high fraction of targets without spectroscopically determined redshifts. For instance, in one of the most studied samples, the 1 Jy sample (Stickel et al. 1991), 23 of 37 targets have a spectroscopically secured redshift with the remaining being unknown, lower limits from an absorption line system or uncertain due to a detection of a single line (Rector \& Stocke 2001). Large ( $8 \mathrm{~m}$ and up) telescopes are often needed to secure the redshift (e.g. Sbarufatti et al. 2009), but even then there often remains a fraction of targets with unknown redshifts.

Sbarufatti et al. (2005) discussed in detail the method of estimating the redshift of BL Lacs through detection of their host galaxies. They showed that the absolute magnitude distribution of BL Lac host galaxies is roughly Gaussian with an average of $M_{\mathrm{R}}^{\text {host }}=-22.8$ and $\sigma=0.5 \mathrm{mag}$. This opens the possibility of

* Based on observations made with the Nordic Optical Telescope, operated on the island of La Palma jointly by Denmark, Finland, Iceland, Norway, and Sweden, in the Spanish Observatorio del Roque de los Muchachos of the Instituto de Astrofisica de Canarias. estimating the redshift using the host galaxy as a "standard candle" if sufficiently deep optical or near-infrared images can be obtained. This method has been applied to several BL Lacs to estimate their redshifts (Nilsson et al. 2008; Meisner \& Romani 2010). Given the width of the host galaxy luminosity distribution, the imaging redshifts are not as accurate as spectroscopic redshifts. The accuracy of the method is \pm 0.05 in redshift, if high signal-to-noise data are available, but in practice the redshift error is usually larger due to noise in derived host galaxy properties (Sbarufatti et al. 2005).

In this paper we report the results of deep optical imaging of two $\gamma$-ray bright BL Lacs, RGB 0136+391 and PKS 0735+178, which were detected by the Fermi-LAT already during first three months of operations (Abdo et al. 2009). For $\gamma$-ray emitting BL Lac objects the redshift estimation is particularly important and there are large dedicated spectroscopy programs for determining redshift for Fermi-LAT detected active galactic nuclei. Still $56 \%$ of the LAT detected BL Lac objects have no redshift measurement (Ackermann et al. 2011), which makes population studies difficult. RGB $0136+391$ is particularly interesting, because of its very hard $\gamma$-ray spectrum (spectral index 1.69) in the Fermi-LAT range and the very recent discovery of very-highenergy (VHE) $\gamma$-rays from the source (Mazin, in prep.). VHE $\gamma$-rays do not travel unaffected in the intergalactic space, but are absorbed by the extragalactic background light (EBL). The EBL leaves an imprint on the observed energy spectrum and if the redshift of the emitting object is known, the observed energy spectrum can be used to set constrains to density and evolution of EBL with redshift (e.g. Stecker et al. 1996; Aharonian et al. 2006; Mazin \& Raue 2007; Meyer et al. 2012). Alternatively, if the redshift is not known the observed VHE $\gamma$-ray spectrum 
combined with the $\mathrm{GeV}$ range spectra can also be used to derive independent estimation for the redshift of the object (Prandini et al. 2010, 2011).

RGB 0136+391 was first identified as a BL Lac object by Laurent-Muehleisen et al. (1998) who saw a featureless optical spectrum. Following attempts (Wei et al. 1999; Bauer et al. 2000; Caccianiga et al. 2002; Piranomonte et al. 2007) have all failed to detect any spectral features and the redshift remains unknown. Piranomonte et al. (2007) derived a lower limit $z>0.193$ based on the absence of host galaxy features in the spectrum. In the only imaging attempt so far, Nilsson et al. (2003) did not detect the host galaxy in a $1200 \mathrm{~s} R$-band exposure.

PKS $0735+178$ was classified and a BL Lac object by Carswell et al. (1974), who detected no emission lines, but a strong $\lambda 2798 \AA$ MgII absorption system at $z=0.424$ providing a lower limit for the redshift. Later spectroscopy (e.g. Stickel et al. 1993; Falomo et al. 1994; Rector \& Stocke 2001) has confirmed the absorption system, but no emission features or host galaxy absorption lines have been detected and the redshift remains unknown. Optical imaging (Hutchings et al. 1988; Abraham et al. 1991; Stickel et al. 1993; Falomo \& Ulrich 2000; Scarpa et al. 2000; Pursimo et al. 2002) has also failed to detect the host galaxy. Scarpa et al. (2000) give a lower limit $R>20.44$ for the host galaxy based on a 440 s HST image through the $F 702 \mathrm{~W}$ filter.

Despite of the strong (equivalent width $W_{\text {obs }} \sim 3.6 \AA$, Rector $\&$ Stocke 2001) MgII absorption line, the absorbing galaxy has remained hidden. Hutchings et al. (1988) reported a bridge of $R$-band emission from PKS $0735+178$ towards the galaxy 7 $\operatorname{arcsec}$ NW with a SB of 26.0-25.2 mag/sq. arcsec. Later imaging by Stickel et al. (1993) did not see this bridge and the galaxy $7 \operatorname{arcsec} \mathrm{NW}$ was measured to have $z=0.645$. There are two possible detections of the absorbing galaxy: Pursimo et al. (1999) reported a faint $(R=21.9)$ elongated structure 3 arcsec NE of PKS $0735+178$ and Falomo \& Ulrich (2000) saw faint emission 3.5 arcsec $E$ of $0735+178$ suggesting it could be the intervening $z=0.424$ system, although they couldn't rule out the possibility that it is just a background target.

The brightness of the BL Lac nucleus relative to the host galaxy often makes it very difficult to determine the host galaxy parameters (magnitude and effective radius) accurately or to detect the host galaxy in the first place. Given that the nucleus is often highly variable, it is advantageous to wait for the moment when it is in a low state. We are currently performing optical monitoring of $\sim 60$ blazars, including RGB $0136+391$ and PKS $0735+178$ (see $^{1}$ and e.g. Aleksić et al. 2011, for results of this monitoring). We are running a parallel program at the Nordic Optical Telescope (NOT) to perform deep I-band imaging of a sample of gamma-ray emitting blazars or potential gamma-ray emitters whenever our monitoring shows that the optical brightness has fallen below a pre-defined threshold. Instead of observing the whole sample at once we wait for the optimal moment. To improve the chances of detecting the host galaxy the threshold level is chosen to represent a very low optical level of each target considering the historical light curve.

In December 2008 the $R$-band brightness of RGB 0136+391 was observed to be close to the lowest level since the beginning of our monitoring (fall 2002) and in January 2011 the brightness of PKS $0735+178$ reached $R=16.7$, a very low level considering the long-term historical light curve (Ciprini et al. 2007). Observations were promptly triggered and performed at the NOT and the results are reported in this paper.

\footnotetext{
1 http://users.utu.fi/kani/1m/index.html
}

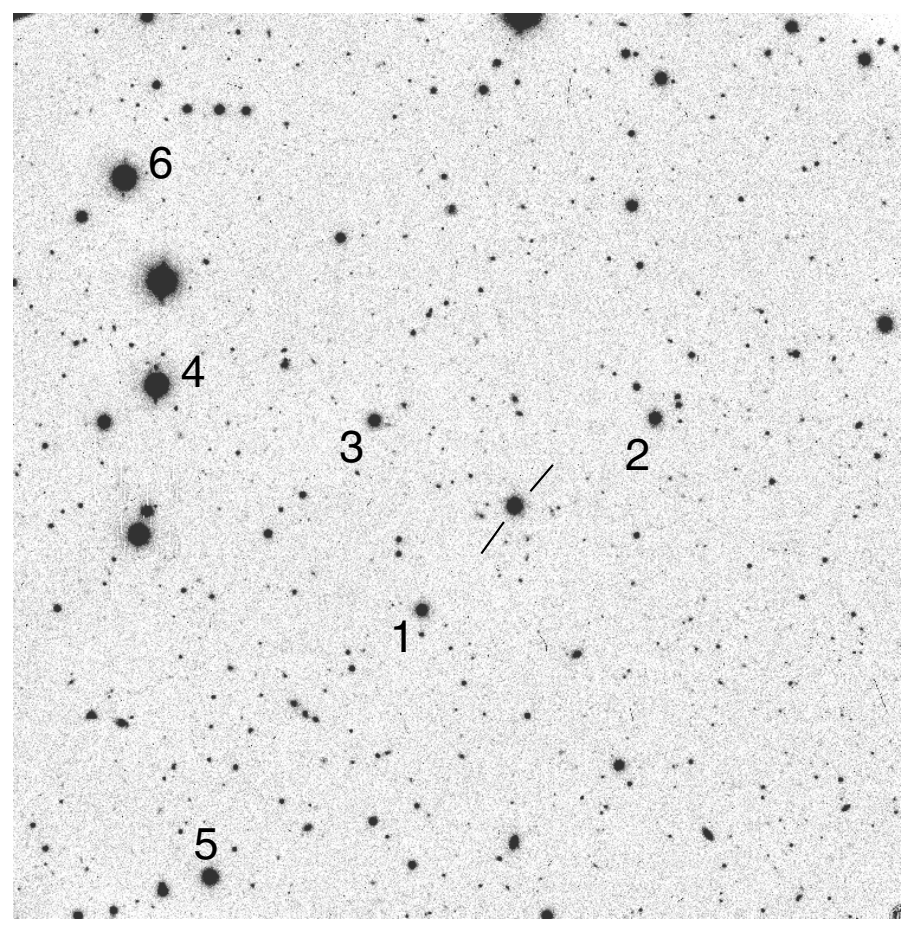

Fig. 1. Summed image of RGB $0136+391$ with the stars discussed in the text labeled. Field size is $5.7 \times 5.8$ arcmin. North us up and east is to the left.

Throughout this paper we use the cosmology $H_{0}=$ $70 \mathrm{~km} \mathrm{~s}^{-1} \mathrm{Mpc}^{-1}, \Omega_{\mathrm{M}}=0.3$ and $\Omega_{\Lambda}=0.7$.

\section{Observations and data reduction}

RGB 0136+391 was observed at the Nordic Optical Telescope (NOT) through an $I$-band filter with almost uniform transmission between 725 and $825 \mathrm{~nm}$ on December 7th, 2008. We used the ALFOSC camera with a gain of $0.726 \mathrm{e}^{-} / \mathrm{ADU}$ and readout noise of $4.2 \mathrm{e}^{-}$. The field of view of the $2048^{2} \mathrm{E} 2 \mathrm{~V}$ chip was $\sim 6.5 \times 6.5 \operatorname{arcmin}(0.189 \mathrm{arcsec} / \mathrm{pixel})$. Altogether 131 images with individual exposure times between $25-100 \mathrm{~s}$ were obtained resulting in a total exposure time of $3830 \mathrm{~s}$. In order to make a fringe correction image the telescope was moved between each exposure. The images were bias-subtracted and flat-fielded with twilight flats and a fringe correction image was constructed from the exposures and subtracted. The images were then registered using 15 unsaturated stars in the field and coadded. The summed image shown in Fig. 1 has a full width at half maximum (FWHM) of 0.61 arcsec. Due to the presence of a quarter Moon in the sky the background brightness was $19.2 \mathrm{mag} / \mathrm{sq}$. arcsec, $\sim 0.8$ mag brighter than average dark sky on La Palma ${ }^{2}$. The field was calibrated using $I$-band observations made with the $35 \mathrm{~cm}$ KVA (Kungliga Vetenskapsakademien) telescope on La Palma in photometric conditions on Jan 11th, 2012. The standard star field PG 0231+055 (Landolt 1992) was observed at the same airmass as the RGB $0136+391$ field and the $I$-band magnitudes of stars 1-6 were derived from these observations. The same stars were then used to determine the zero point of our summed image. The error of the derived zero point is $0.02 \mathrm{mag}$, excluding color effects (see below).

PKS $0735+178$ was observed with the same instrumental setup at the NOT on January 18th, 2011. Altogether 55 images

2 La Palma technical note 115, http://www.ing.iac.es/ Astronomy/observing/conditions/skybr/skybr.html 


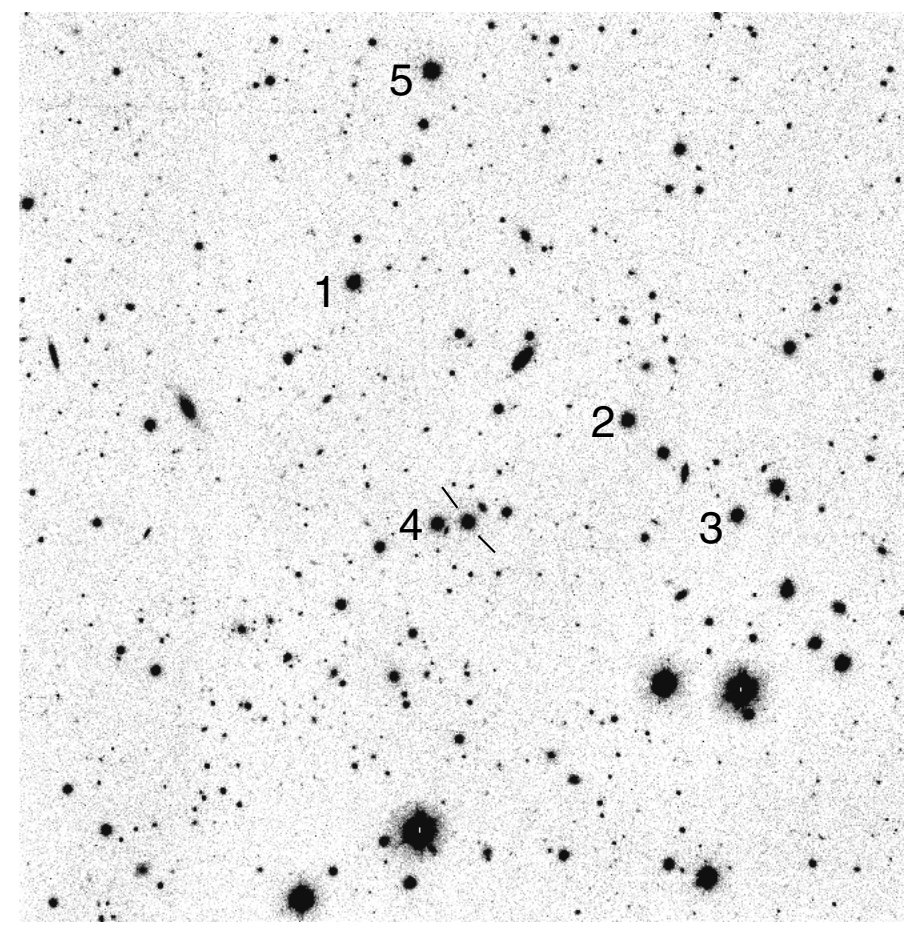

Fig. 2. Summed image of PKS $0735+178$ with the stars discussed in the text labeled. Field size is $5.2 \times 5.4$ arcmin. North us up and east is to the left.

with exposure times of 150-300 s were obtained. The images were processed in a way identical to RGB $0136+391$. The final coadded image shown in Fig. 2 has a total exposure time of $10250 \mathrm{~s}$ and a FWHM of $0.60 \mathrm{arcsec}$. The observations were influenced by almost full ( 90\%) Moon 21 degrees away from PKS $0735+178$. As a result the sky background is relatively high (17.7 mag/sq. arcsec) and there is scattered light present in the raw images resulting in an uneven background. The fringe removal procedure mostly eliminates the scattered light but leaves a small $(0.1 \%$ peak-to-peak amplitude) undulation of the background at large spatial scales, barely visible in Fig. 2. Therefore, the background of PKS $0735+178$ and its point spread function (PSF) stars was subtracted by fitting a two-dimensional linear slope to the sky pixels surrounding the targets, effectively removing any remaining tilt in the background. Calibration of the field was done through star 5, for which Ciprini et al. (2007) give $I=15.12 \pm 0.06$. This star was unsaturated in several exposures, which were used to derive I-band magnitudes for stars 1-3, which were then used to determine the zero point of the coadded image.

Since the transmission curve of the filter used here closely matches the I-band transmission (Bessell 1990), the color effects are expected to be small. According to the NOT zero point monitoring pages ${ }^{3}$ the $(V-I)$ color term is -0.03 . Two of the stars used for calibration, star 4 in RGB $0136+391$ and star 5 in PKS 0735+178, have $V-I \approx 0.8$ (Nilsson et al. 2007; Ciprini et al. 2007, present work). Assuming the rest of the stars used for calibration have similar colors and that the host galaxy $V-I=2.6$ (elliptical galaxy color at $z=0.5$, Fukugita et al. $1995)$ we derive the error of host galaxy magnitudes due to filter mismatch to be $\approx 0.05 \mathrm{mag}$.

\footnotetext{
3 http://www.not.iac.es/instruments/alfosc/zpmon/
}

\section{Analysis}

We searched for evidence of the host galaxy by fitting twodimensional models to the observed image. The detections are further tested by simulations, which are also used to derive error bars for the derived parameters.

The fitting procedure is the same as employed in our previous studies (e.g. Nilsson et al. 1999, 2008). In short, the twodimensional model consists of two components, the unresolved core and a host galaxy whose surface brightness profile follows the de Vaucouleurs law $(\beta=1 / n=0.25$, where $n$ is the profile index from Sersic 1968). The model is described by five parameters: location $x-y$, assumed to be the same for the core and the host galaxy, core magnitude $I_{\text {core }}$, host galaxy magnitude $I_{\text {host }}$ and host galaxy effective (half light) radius $r_{\text {eff }}$. Since the host galaxies were known from previous imaging to be very faint, the ellipticity and position angle were kept at constant value $=0$. We used a Levenberg-Marquardt loop to find the set of parameters which minimized the $\chi^{2}$ between the data and the model. The models were convolved with an empirical PSF derived from stars in the vicinity of the target. In the case of RGB $0136+391$ we used stars $1-4$ and in the case of PKS $0735+178$ stars $1-3$. The fitting proceeded in two phases. First we fitted a model consisting of an unresolved component only, i.e. the model had three free parameters $\left(x, y\right.$ and $\left.I_{\text {core }}\right)$. After this the position was fixed and the full core + host galaxy model was fit with $I_{\text {core }}, I_{\text {host }}$ and $r_{\text {eff }}$ as free parameters.

If the host galaxy is below detection limit these fits usually converge towards $r_{\text {eff }} \rightarrow 0$ or $r_{\text {eff }} \rightarrow \infty$. In case we found $r_{\text {eff }}>0$ we tested the significance of the detection by performing fits to simulated data. We created a set of $\sim 100$ simulated images of the target using the fitted core and host galaxy values. The $\beta$ value for the surface brightness profile of the simulated galaxy was drawn from a Gaussian distribution with average equal to 0.25 and $\sigma=0.025$. The fitting, however, was made with fixed $\beta=0.25$. Each model was convolved with a PSF consisting of a Moffat $\beta=2.5$ profile with a FWHM equal to the observed one, an ellipticity randomly drawn from an uniform distribution between 0.0 and 0.06 and a position angle drawn from an uniform distribution between 0.0 and 180 degrees. To these images we added Gaussian noise representing photon and readout noise, scaled to the same level as in the observed data and a random offset drawn from a Gaussian distribution with $\sigma=\sigma_{\text {sky }} / 30$, where $\sigma_{\text {sky }}$ is the sky rms scatter, representing the error made in sky level estimation.

For each simulation we also constructed a second PSF, which slightly deviated from the PSF used in convolving the model. The ellipticity of the second PSF was drawn from an uniform distribution with mean equal to the ellipticity of the first PSF and maximum deviation of 0.02. Similarly, the position angle deviated maximum 10 degrees from the position angle of the first PSF. To this second PSF we also added random photon and readout noise plus an offset the simulate the sky determination error. The second PSF was then used in the simulated fits as the assumed PSF. Using a different PSF for the model creation and for the fitting enabled us to roughly simulate the PSF variation seen in the images and to produce residuals which qualitatively resemble the residuals seen in actual images, although the latter tend to be a bit more complex than what these simple simulations can produce. The limits to the distributions above were selected to match the residuals quantitatively, i.e. the peak-to-peak residuals in the simulations were on average equal to the real residuals. Similarly, the average $\chi^{2}$ in the simulations was close to the $\chi^{2}$ of the fit to the real data. 


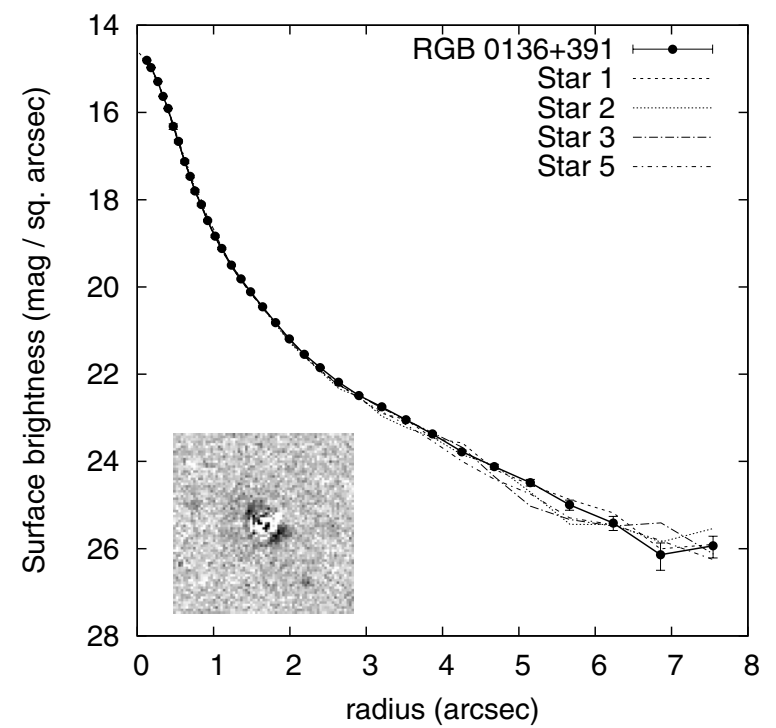

Fig. 3. I-band surface brightness profile of RGB 0136+391 (thick solid line) and four stars in the field. The stars are labeled in Fig. 1. Insert: residuals after subtracting the PSF from RGB $0136+391$. Field size is $14 \times 14$ arcsec. The error bars include contribution from the empirical scatter in each bin and the error in sky background determination.

After the simulations we computed the standard deviation of the host galaxy magnitudes $\sigma_{\text {host }}$ and the range of effective radii which includes $67 \%$ of the simulated values. We didn't use standard deviation for the effective radius because the distribution was asymmetric with a longer tail towards high values. To consider a host galaxy detected we required $\sigma_{\text {host }}<0.3 \mathrm{mag}$ and that the median effective radius of the simulations is $>2 \sigma_{\text {reff }}$, where $\sigma_{\text {reff }}$ is the lower $67 \%$ error bar. The more relaxed criterion for $r_{\text {eff }}$ was used because $r_{\text {eff }}$ is generally more difficult to constrain and requiring $r_{\text {eff }}>3 \sigma_{\text {reff }}$ would exclude host galaxies whose surface brightness profile clearly exceeds any reasonable PSF variability.

\section{Results}

\subsection{RGB $0136+391$}

Figure 3 shows the surface brightness (SB) profile of RGB $0136+391$ together with the profiles of four stars in the FOV. The profiles of the stars have been scaled to the same total flux as RGB $0136+391$. The SB profile of RGB $0136+391$ is totally consistent with a point source, with no excess in the outer part of the profile to indicate the presence of a host galaxy. Examination of the two-dimensional residuals (Fig. 3, insert) yields the same conclusion: no obvious signs of the host galaxy above PSF residuals can be seen. The model fits confirm this conclusion: the final values returned by the fits are $I_{\text {core }}=15.20 \pm 0.01, I_{\text {host }}=19.46 \pm 0.32$ and $r_{\text {eff }}=1.5_{-0.8}^{+2.5} \mathrm{arcsec}$, where the error bars come from the simulations described above. This target thus remains unresolved.

We then derived a lower limit for the redshift of RGB 0136+391 using the following procedure: if the distribution of BL Lac host galaxy magnitudes is as shown by Sbarufatti et al. (2005), then $95 \%$ of BL Lac host galaxies are brighter than $M_{R}=-22.0$. We thus created 100 simulated BL Lac objects at $z=0.25,0.30, \ldots, 0.45$ with core magnitude $I_{\text {core }}=15.20$ and $I_{\text {host }}$ and $r_{\text {eff }}$ corresponding to $M_{\mathrm{R}}^{\text {host }}=-22.0$ and $R_{\text {eff }}=10 \mathrm{kpc}$. We first computed the absolute $I$-band magnitude $M_{I}$ using

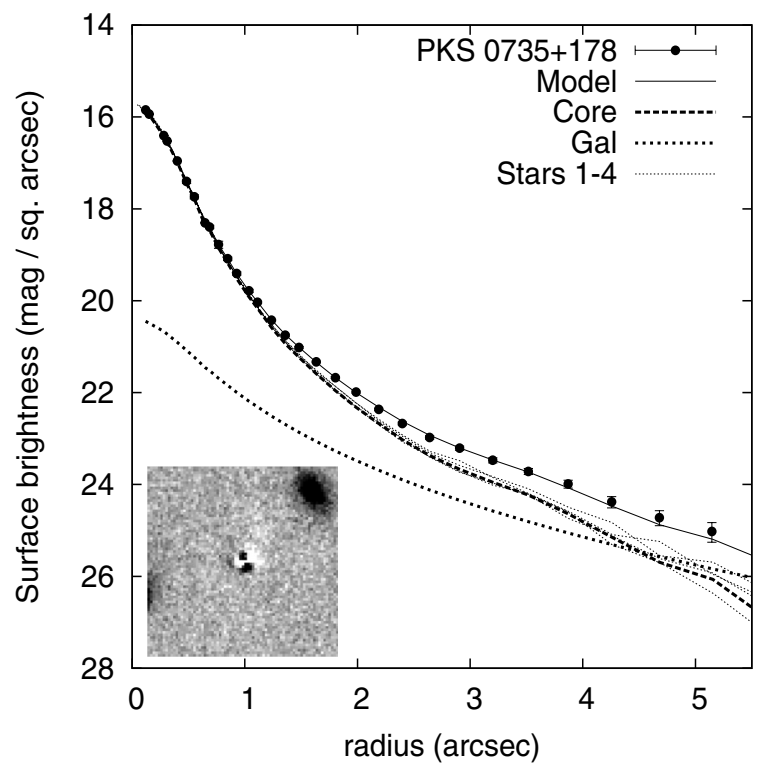

Fig. 4. Surface brightness decomposition of PKS 0735+178. In addition to PKS $0735+178$ and model surface brightnesses, the profiles of stars 1-4 are shown. Insert: the residuals after subtracting the core + host galaxy model. Field size is $14.2 \times 14.2$ arcsec.

$R-I=0.70$ (Fukugita et al. 1995) and the apparent $I$-band magnitude $m_{I}$ from

$m_{I}=M_{I}+D M+K_{I}+A_{I}-E(z)$

where $D M$ is the distance modulus, $K_{I}$ is the $I$-band K-correction from Fukugita et al. (1995), $A_{I}=0.15$ is the galactic extinction (Schlegel et al. 1998) and $E(z)=0.84 * z$ is the evolution correction, computed using the PEGASE code (Fioc \& Rocca-Volmerange 1997) by assuming galaxy formation $11 \mathrm{Gyr}$ ago and passive evolution thereafter.

At each redshift we then fitted the 100 model images using the same procedure as in the error simulations and computed the standard deviation of $I_{\text {host }}$ and $r_{\text {eff }}$. The result of these simulations was that at $z=0.45\left(m_{I}=19.52\right)$ the host galaxy became unresolved using our criteria. Thus at $z \leq 0.40$ we would have detected the host galaxy with $>95 \%$ probability and thus assign a conservative lower limit $z>0.40$ for RGB $0136+391$.

\subsection{PKS $0735+178$}

Figure 4 shows the SB profile of PKS $0735+178$ together with stars 1-4. The stellar profiles have been again scaled to the same total flux as PKS $0735+178$. Contrary to RGB $0136+391$, there is a clear excess over the stellar profiles. Model fits yield $I_{\text {core }}=16.19 \pm 0.01, I_{\text {host }}=18.64 \pm 0.07$ and $r_{\text {eff }}=1.8 \pm 0.4$ arcsec meeting our criteria for successful detection. Adding the error from zero point determination and filter mismatch the error of $I_{\text {host }}$ becomes 0.11 mag. Given the clear excess and the fit results we conclude that we have detected the host galaxy or a foreground galaxy (see Sect. 5).

The error bars from the simulations do not take into account possible systematic errors, which are difficult to predict and/or quantify. We have tested the sensitivity of our result with respect to our particular choice of software by performing the fits with the GALFIT software (Peng et al. 2002). We used the same image, PSF and sky background as before with the only difference being the software. The result from this fit was $I_{\text {host }}=18.57$ and $r_{\mathrm{eff}}=2.0 \mathrm{arcsec}$, i.e. the host galaxy magnitude differs by $7 \%$ 


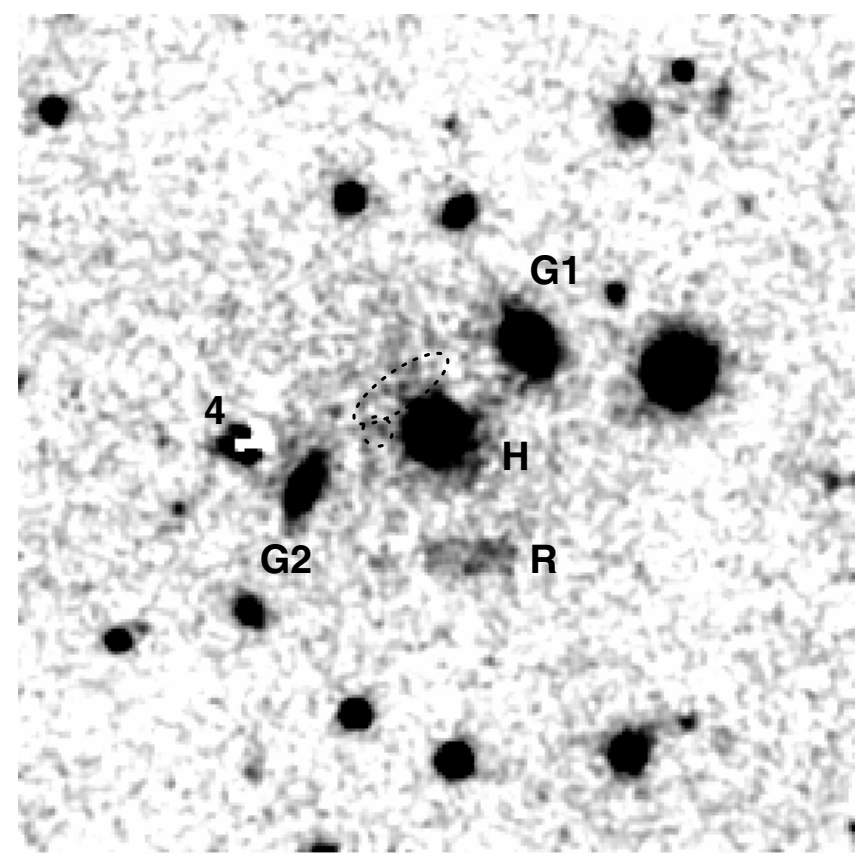

Fig. 5. $47.4 \times 47.4$ arcsec field around PKS $0735+178$ after subtracting the scaled PSF from PKS $0735+178$ and star 4. The image has been smoothed with a Gaussian kernel with $\sigma=1.0$ pix. Galaxies G1 and $\mathrm{G} 2$ and the rectangular test feature $\mathrm{R}$ are discussed in the text. $\mathrm{H}$ is the host galaxy of PKS $0735+178$. The dotted ellipse and circle mark the location of the features discussed by Pursimo et al. (1999) and Falomo \& Ulrich (2000), respectively.

and the effective radius by $11 \%$ from our result. The two programs thus provide very consistent results and we do not expect the systematic errors due to software choice to dominate over statistical noise.

Assuming that the detected galaxy is the host galaxy we estimated the redshift of PKS $0735+178$ using the results of Sbarufatti et al. (2005). Using Eq. (1) with $A_{I}=0.068$ (Schlegel et al. 1998) and $M_{I}=-22.8$ we iteratively determined the the redshift consistent with the observed $I$-band magnitude to be $z=0.45 \pm 0.06$, where the error bar includes the contribution from host magnitude error and the uncertainty of the method itself.

\section{Discussion of PKS $0735+178$}

Scarpa et al. (2000) derived an upper limit $m_{R}>20.44$ for the host galaxy, which together with our result implies $R-I>1.7$. This is redder than the expected $R-I=1.2$ for ellipticals at $z=0.5$ (Fukugita et al. 1995), possibly an indication of systematic differences between the two studies, or of dust reddening in the host galaxy. If significant dust absorption is present in PKS 0735+178, our redshift estimate is too high.

Using the $I$-band data obtained here and previous $R$-band data we now discuss the identity of the MgII absorber. Galaxy G1 in Fig. 5 can be directly ruled out due to the redshift $z=0.645$ measured by Stickel et al. (1993). Pursimo et al. (1999) and Falomo \& Ulrich (2000) detected weak $R$-band emission 3".0 NE and 3.'5 E of PKS 0735+178, respectively, and discussed the possibility of these features being the MgII absorber. Neither of these emission features are visible in our PSFsubtracted I-band image (Fig. 5). Since our images were obtained in bright moonlight and thus not optimal for detecting faint diffuse emission, we tested our sensitivity with the following procedure.

Firstly, since Falomo \& Ulrich (2000) do not give a magnitude for the feature they detected, we cannot estimate if it was detectable in our image. We therefore concentrate on the elongated feature reported 3.'0 NE of PKS $0735+178$ by Pursimo et al. (1999). To roughly simulate this feature we created a rectangular test object marked with $\mathrm{R}$ in Fig. 5. Note that we did not create this feature at the correct location to better compare with the actual image. The feature has a uniform $I$-band surface brightness of $24.3 \mathrm{mag} / \mathrm{sq}$. arcsec and its dimensions and integrated magnitude 21.9 correspond to those of the elongated featured reported by Pursimo et al. (1999). The test feature is just visible over the background noise and thus we should have detected the Pursimo et al. (1999) feature in our image if its $R-I$ color $>0$. Since the range of $R-I$ colors of $\mathrm{E}$ to Im galaxies at $z=0.424$ is expected to be form 1.1 to 0.4 , respectively (Fukugita et al. 1995), we conclude that we should have detected the Pursimo et al. (1999) feature, if its colors are within the range of "normal" galaxies. Given the non-detection in our I-band image the Pursimo et al. (1999) feature is unlike to be the absorber. It could be a line emitting cloud whose emission wavelength falls into the $R$-band but outside the $I$-band. Of the lines bluewards of the $R$-band, $\lambda 5007$ [OIII] is the most likely candidate. This line would place the cloud at $z=0.13-0.44$ when $50 \%$ transmission limits of the $R$-band filter are considered.

Since we do no detect the feature discussed by Pursimo et al. (1999) and the detection probability of the Falomo \& Ulrich (2000) feature cannot be estimated we turn our attention to galaxy G2. This galaxy lies at projected distance of 8.'2 (46 kpc) from PKS 0735+178. Given the rest frame equivalent width of the MgII line in PKS 0735+178, $W_{0}=3.6 \AA /\left(1+z_{\text {abs }}\right)=2.5 \AA$, we ask if this galaxy could be the absorber. For comparison we use Zibetti et al. (2007), who studied $2844 \mathrm{MgII}$ absorbers with $W_{0}>0.8 \AA$ and $0.37<z<1$ using the SDSS DR4. We first note that $W_{0}=2.5 \AA$ implies very strong absorption: $93 \%$ of the systems studied by Zibetti et al. (2007) have $W_{0}<2.5 \AA$. If we concentrate on the relevant section of the parameter space in Zibetti et al. (2007), $0.37<z<0.55$ and $W_{0}>1.58 \AA$, we note that $\mathrm{MgII}$ absorbers can be found up to projected distances of $\sim 100 \mathrm{kpc}$ from the background source. In this section of the parameter space the probability of finding an $\mathrm{MgII}$ absorber at projected distance of $46 \mathrm{kpc}$ is $\sim 25 \%$ (see Fig. 9 in Zibetti et al. 2007). Given that the probability of finding an Mg II absorber at a given distance decreases with $W_{0}$ and the $W_{0}$ of the MgII line in PKS $0735+178$ is at the high end of the distribution, the probability of $\mathrm{G} 2$ being the absorber is probably considerably lower. The $I$-band magnitude of G2 is $20.4 \pm 0.1$ which gives $R-I=0.5$ when combined with the $R$-band magnitude in Pursimo et al. (1999). This color is consistent with a late type (Scd) galaxy at $z=0.424$. The absolute magnitude of $\mathrm{G} 2$ at $z=0.424$ would be $M_{R}=-20.9 \approx M_{R}^{*}+0.3$. In the light of these results $\mathrm{G} 2$ cannot be completely ruled out as the absorbing galaxy, although finding such a system is relatively unlikely.

As the next option we consider the possibility that the detected host galaxy is actually a foreground object and not the real host galaxy. If this is the case, then the impact parameter must be very close to zero since the detected galaxy appears to be well centered on the BL Lac nucleus. To check for possible offsets between the core and the host galaxy we performed the host galaxy fit with 7 free parameters, i.e. in addition to $I_{\text {core }}, I_{\text {host }}$ and $r_{\text {eff }}$ also the $x-y$ positions of the core and the host galaxy were allowed to change freely. The core-host galaxy offset was 0.67 pixels 
$(0.13 \mathrm{arcsec})$ in this case, indicating that the core is well centered on the host galaxy. Such a system is capable of producing gravitational lensing phenomena, but Rector \& Stocke (2003) did not detect any signs of gravitational macrolensing (Einstein rings or multiple images) in their 8.46 and $14.94 \mathrm{GHz}$ maps with $\sim 0.2$ arcsec resolution. Furthermore, the result of Zibetti et al. (2007) indicate that strong absorbers are associated with galaxies with intense star forming, which should be visible in the optical spectrum as narrow emission lines. These findings do not disprove the hypothesis that the detected galaxy is an intervening system with a small impact parameter, but they make it less attractive with respect to the alternative, that we have detected the actual host galaxy.

Lastly we note that the derived redshift $0.45 \pm 0.06$ is within errors equal to the absorption redshift 0.424 . It could thus be possible that the absorption occurs in the host galaxy of PKS $0735+178$ itself. This would imply the PKS $0735+178$ host galaxy to be a gas-rich system since strong $\mathrm{Mg}$ II absorption is often associated with damped Lyman-alpha systems (DLAs) with high HI gas content (Péroux et al. 2004). Further observations are clearly needed in order to determine which, if any, of the above scenarios is the correct one. A spectrum of G2 is needed in order to see if its redshift is compatible with being the absorbing galaxy. An ultraviolet spectrum of PKS 0735+178 detecting the Lyman-alpha line would also shed new light on the nature of the absorber by measuring of the HI column density.

\section{Conclusions}

We have presented deep I-band imaging of two BL Lacertae objects, RGB 0136+391 and PKS 0735+178, obtained during a deep minimum in the optical light curve. Despite of a deep exposure obtained under excellent seeing conditions $\left(0{ }^{\prime} 6\right)$ the host galaxy of RGB $0136+391$ remains unresolved. We derive $z>0.40$ for RGB $0136+391$ using simulated host galaxies at different redshifts with $M_{R}=-22.0$ and $R_{\text {eff }}=10 \mathrm{kpc}$. This makes RGB $0136+391$ one of the most distant VHE $\gamma$-ray sources detected up to date.

The surface brightness profile of PKS 0735+178 reveals an excess over a point source, which, if fitted by a de Vaucouleurs profile, corresponds to a galaxy with $I=18.64 \pm 0.11$ and $r_{\mathrm{eff}}=$ $1.8 \pm 0.4$ arcsec. Under the assumption that BL Lac host galaxies can be used as standard candles (Sbarufatti et al. 2005) we derive $z=0.45 \pm 0.06$ for PKS $0735+178$.

We also discuss the identity of the galaxy responsible for the strong MgII absorption line in PKS 0735+178 at $z_{\mathrm{abs}}=0.424$ using previous $R$-band imaging and the $I$-band data in this paper. We do not detect the two faint objects suggested earlier to be responsible for the absorption or any other obvious candidates in the vicinity of PKS $0735+178$. We identify a galaxy $8 . ! 2$ of PKS $0735+178$ (46 kpc projected distance) as a candidate for the absorber, although the probability of strong absorption $\left(W_{0}=\right.$ $2.5 \AA$ ) at this projected distance is very low. Further observations are needed in order to reveal the identity of the absorber towards PKS 0735+178.
Acknowledgements. The data presented here were obtained with ALFOSC, which is provided by the Instituto de Astrofisica de Andalucia (IAA) under a joint agreement with the University of Copenhagen and NOTSA. This research has made use of NASA's Astrophysics Data System Bibliographic Services. This research has made use of NASA's Astrophysics Data System Bibliographic Services. This research has made use of the NASA/IPAC Extragalactic Database (NED) which is operated by the Jet Propulsion Laboratory, California Institute of Technology, under contract with the National Aeronautics and Space Administration.

\section{References}

Abdo, A. A., Ackermann, M., Ajello, M., et al. 2009, ApJ, 700, 597 Abraham, R. G., Crawford, C. S., \& McHardy, I. M. 1991, MNRAS, 252, 482 Ackermann, M., Ajello, M., Allafort, A., et al. 2011, ApJ, 743, 171 Aharonian, F., Akhperjanian, A. G., Bazer-Bachi, A. R., et al. 2006, Nature, 440, 1018

Aleksić, J., Antonelli, L. A., Antoranz, P., et al. 2011, A\&A, 530, A4

Bauer, F. E., Condon, J. J., Thuan, T. X., \& Broderick, J. J. 2000, ApJS, 129, 547 Bessell, M. S. 1990, PASP, 102, 1181

Caccianiga, A., Marchã, M. J., Antón, S., Mack, K.-H., \& Neeser, M. J. 2002, MNRAS, 329, 877

Carswell, R. F., Strittmatter, P. A., Williams, R. E., Kinman, T. D., \& Serkowski, K. 1974, ApJ, 190, L101

Ciprini, S., Takalo, L. O., Tosti, G., et al. 2007, A\&A, 467, 465

Falomo, R., \& Ulrich, M.-H. 2000, A\&A, 357, 91

Falomo, R., Scarpa, R., \& Bersanelli, M. 1994, ApJS, 93, 125

Fioc, M., \& Rocca-Volmerange, B. 1997, A\&A, 326, 950

Fukugita, M., Shimasaku, K., \& Ichikawa, T. 1995, PASP, 107, 945

Hutchings, J. B., Johnson, I., \& Pyke, R. 1988, ApJS, 66, 361

Landolt, A. U. 1992, AJ, 104, 340

Laurent-Muehleisen, S. A., Kollgaard, R. I., Ciardullo, R., et al. 1998, ApJS, 118,127

Mazin, D., \& Raue, M. 2007, A\&A, 471, 439

Meisner, A. M., \& Romani, R. W. 2010, ApJ, 712, 14

Meyer, M., Raue, M., Mazin, D., \& Horns, D. 2012, A\&A, 542, A59

Nilsson, K., Pursimo, T., Takalo, L. O., et al. 1999, PASP, 111, 1223

Nilsson, K., Pursimo, T., Heidt, J., et al. 2003, A\&A, 400, 95

Nilsson, K., Pasanen, M., Takalo, L. O., et al. 2007, A\&A, 475, 199

Nilsson, K., Pursimo, T., Sillanpää, A., Takalo, L. O., \& Lindfors, E. 2008, A\&A, 487, L29

Peng, C. Y., Ho, L. C., Impey, C. D., \& Rix, H.-W. 2002, AJ, 124, 266

Péroux, C., Deharveng, J.-M., Le Brun, V., \& Cristiani, S. 2004, MNRAS, 352, 1291

Piranomonte, S., Perri, M., Giommi, P., Landt, H., \& Padovani, P. 2007, A\&A, 470, 787

Prandini, E., Bonnoli, G., Maraschi, L., Mariotti, M., \& Tavecchio, F. 2010, MNRAS, 405, L76

Prandini, E., Mariotti, M., \& Tavecchio, F. 2011 [arXiv: 1111. 0913]

Pursimo, T., Nilsson, K., Sillanpää, A., Takalo, L. O., \& Heidt, J. 1999, in BL Lac Phenomenon, eds. L. O. Takalo, \& A. Sillanpää, ASP Conf. Ser., 159, 385

Pursimo, T., Nilsson, K., Takalo, L. O., et al. 2002, A\&A, 381, 810

Rector, T. A., \& Stocke, J. T. 2001, AJ, 122, 565

Rector, T. A., \& Stocke, J. T. 2003, AJ, 125, 2447

Sbarufatti, B., Treves, A., \& Falomo, R. 2005, ApJ, 635, 173

Sbarufatti, B., Ciprini, S., Kotilainen, J., et al. 2009, AJ, 137, 337

Scarpa, R., Urry, C. M., Falomo, R., Pesce, J. E., \& Treves, A. 2000, ApJ, 532, 740

Schlegel, D. J., Finkbeiner, D. P., \& Davis, M. 1998, ApJ, 500, 525

Sersic, J. L. 1968, Atlas de galaxias australes (Cordoba, Argentina: Observatorio Astronomico)

Stecker, F. W., de Jager, O. C., \& Salamon, M. H. 1996, ApJ, 473, L75

Stickel, M., Padovani, P., Urry, C. M., Fried, J. W., \& Kuehr, H. 1991, ApJ, 374, 431

Stickel, M., Fried, J. W., \& Kuehr, H. 1993, A\&AS, 98, 393

Wei, J. Y., Xu, D. W., Dong, X. Y., \& Hu, J. Y. 1999, A\&AS, 139, 575

Zibetti, S., Ménard, B., Nestor, D. B., et al. 2007, ApJ, 658, 161 